\title{
Prediction of Rubber Aging Life by Intelligent Neural Network
}

\author{
Hong HE ${ }^{\mathrm{a}, 1}$, WenQi HU1 ${ }^{1}$, ShenJun GAO² \\ ${ }^{1}$ Electromechanic Engineering College, Beijing University of Chemical Technology, Chaoyang, Beijing, China \\ ${ }^{2}$ Shanghai Jingyi Rubber Technology Co., Ltd., Shanghai, China
}

\begin{abstract}
Predicting the agin life of rubber is mostly based on traditional dynamic methods. These methods often have some limitations, which can not reflect the influence of certain factors. To avoid such limitations, a BP neural network model was established to predict the aging life of rubber. Comparing with the BP neural network model, results from the genetic algorithm optimization model (GA-BP) and the particle swarm optimization model (PSO-BP) showed that the GA-BP network model has better stability and accuracy and can quickly get the global optimal solution. The prediction accuracy of the GA-BP neural network model is better than that of the traditional dynamic model and its result is in good agreement with the experimental data.
\end{abstract}

\section{Introduction}

The aging of rubber is a very common phenomenon. It is the main form of rubber products failure. Therefore, the annual loss caused by rubber aging is great. Predicting the rubber aging life in advance could provide references time for replacement or maintenance of rubber products and also provide an evaluation tool for research and development of new rubber composite materials.

At present, there are some methods[1-5] to predict the rubber aging life. They are mainly the traditional mathematical function methods. These methods are trying to establish the mathematical function relationship among variables in the rubber aging process. Because of the complexity of the aging process, these mathematical functions often have some limitations. They could not reflect the influence of certain factors. However the neural network method can skip the link of establishing the mathematical function, it could learn the abstract network relationship between the input and output based on the experimental data, and there is no limit to the number of the input and output of the model. In view of this this paper proposed a neural network model to predict the aging life of rubber, based on the BP neural network method and algorithm optimization.

\section{The establishment of a BP neural network prediction model for rubber aging life}

For BP neural networks, a continuous function in any closed interval can be approximated by a single hidden layer in BP network. Although an increase in the number of hidden layers would enhance the processing capacity of the neural network, it would also make the network training more complex[6]. Thus, three layers network was chosen in the predicting model.

The relationship between input layer and output layer can be trained on the basis of rubber accelerated aging experimental samples. Temperature which was as the influence factor of aging and aging properties such as hardness, modulus of tension,etc were used as input layers parameters and the rubber aging life as output parameter, as shown in Figure 1. Here the retention rate of permanent deformation for rubber compression was selected as aging property.

The transfer functions "tansig" and "purelin", between the hidden layer and the output layer were used respectively. In order to accelerate the learning rate, the training function "trainlm" was adopted, whose convergence performance was better.

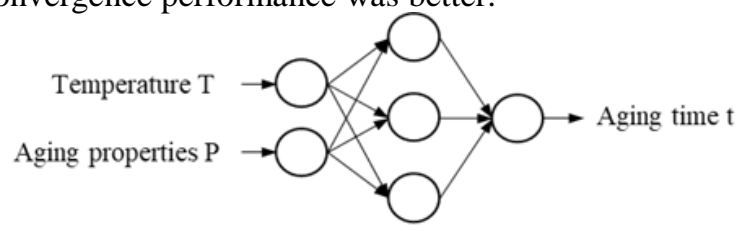

Input layer Hidden layer Output layer

Figure 1 BP neural network prediction model.

In BP neural networks, the selection of the nodes number in the hidden layer is very important. It not only affects the performance of the neural network, but also is one of the main causes of "over fitting"[7]. However, there is not universal method. The range of this number generally was roughly estimated according to the relevant empirical formula. The nodes number in the hidden layer for predicting model was determined to be three and the determination process of optimal number was as follows.

For a single hidden layer neural network, Nielsen proposed the empirical formula: 


$$
m=2 n+1
$$

where $m$ and $n$ are the number of nodes in the hidden layer and input layer respectively.

According to the formula (1), estimating the range of node number in the hidden layer 1 6, then using " cutand-try" to determine the empirical number of hidden layer nodes. Because this number had a very important influence on the generalization ability and prediction accuracy of results, the empirical number of hidden layer nodes can be deduced according to the stability and accuracy of neural network prediction. The variance $(\delta)$ of prediction results was used as a stability index, and the mean value of the mean square error (MSE) between the network calculation result and the expected value as the simulation precision index.

Take the aging of NBR-26 gasket as an example to demonstrate the training process between input layer and output layer. The accelerated aging test data of NBR-26 gasket is derived from literature[8]. Aging temperature were $60,70,80,90$ and $100^{\circ} \mathrm{C}$ respectively. Aging time $\mathrm{t}$ (day) were $0.5,1,2,3,4,5,7,9,12,16,20,25,32,39$, 64.3 and 99.3 respectively. Because the parameters of input and output in the neural networks have different physical meanings and dimension, and the activation function had saturated characteristics, it is necessary to normalize the data to balance the weight of different input components and avoid the saturation area of the activation function. The temperature $\mathrm{T}$ and aging time $\mathrm{t}$ from the test, or parameters in input layer, were pretreated as follows:

$$
\begin{aligned}
& T=T_{o} / 100 \\
& t=\ln \left(t_{o} \times 10\right) / 10
\end{aligned}
$$

Where $T_{0}$ and $t_{0}$ are the original temperature and original aging time, and $T, t$ are pretreated temperature and pretreated aging time respectively.

Predicting the aging time of the gasket at room temperature $\left(25^{\circ} \mathrm{C}\right)$ when the retention rate of the rubber compression set was reduced to 0.3 by running the neural network model 30 times from 1 to 6 nodes in hidden layer separately, and setting the number of iterations to 100 for each run. The results are shown in Table 1 and Figure 2.

Table 1 comparison of prediction stability and a.ccuracy for BP network with 1-6 nodes in hidden layer.

\begin{tabular}{|c|c|c|}
\hline $\begin{array}{c}\text { Hidden layer } \\
\text { nodes number }\end{array}$ & Variance & $\begin{array}{c}\text { The mean of } \\
\text { MSE }\end{array}$ \\
\hline 1 & $7.46 \times 10^{-5}$ & 63.18 \\
\hline 2 & $1.19 \times 10^{5}$ & 7.87 \\
\hline 3 & $3.45 \times 10^{9}$ & 1.78 \\
\hline 4 & $4.80 \times 10^{15}$ & 0.57 \\
\hline 5 & $5.21 \times 10^{54}$ & 0.41 \\
\hline 6 & $5.17 \times 10^{251}$ & 0.42 \\
\hline
\end{tabular}

As shown in Table 1, when the number of nodes was in $1 \sim 3$, the variance of prediction value was relatively small and it increased sharply from the 4th nodes. However, the mean value of MSE decreased obviously when the number of nodes was in $1 \sim 3$, and it is smaller from the 3 rd nodes. It meant that the stability decreased and the precision increased with the increase of the number of hidden layer nodes.

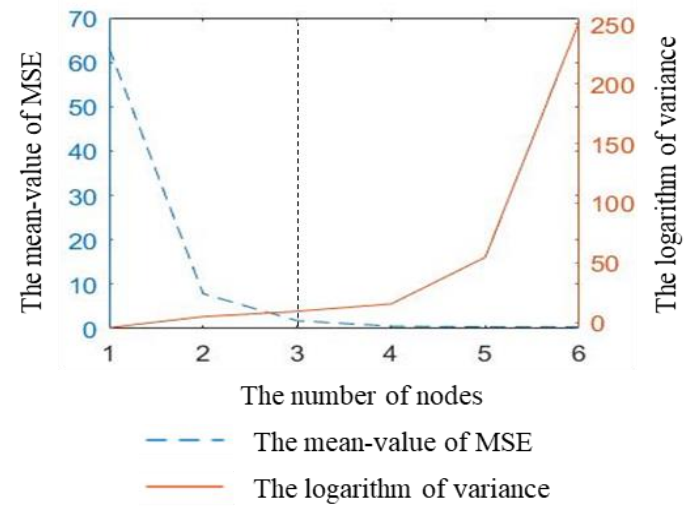

Figure 2 The relation between stability, accuracy of BP neural network and nodes number in the hidden layer.

As shown in Figure 2, selecting 3 nodes for the hidden layer is the best, which not only obtains good stability, but also nice accuracy. The variance from 30 run was still up to $3.45 \times 10^{9}$, that meant the result stability was relatively poor. The cause maybe the weights and thresholds for BP neural network generated randomly. For this reason, the weights and thresholds were optimizted by genetic algorithm and particle swarm optimization, which improve the stability of the BP neural network[9].

\section{The comparison of intelligent algorithms: genetic algorithm and particle swarm optimization}

The parameters of genetic algorithm (GA) and particle swarm optimization (PSO) used are shown in Table 2.

Table 2 The parameters of GA and PSO used.

\begin{tabular}{|c|c|c|c|}
\hline GA & Value & PSO & Value \\
\hline Population size & 20 & Population size & 30 \\
\hline Evolution times & 50 & Evolution times & 50 \\
\hline $\begin{array}{c}\text { Cross } \\
\text { probability }\end{array}$ & 0.4 & Velocity range & {$[-1,1]$} \\
\hline $\begin{array}{c}\text { Mutation } \\
\text { probability }\end{array}$ & 0.1 & Location range & {$[-5,5]$} \\
\hline- & - & $\begin{array}{c}\text { Acceleration } \\
\text { constant }\end{array}$ & 1.49 \\
\hline
\end{tabular}

\subsection{The prediction stability and accuracy of three models}

The genetic algorithm and particle swarm optimization were usded to optimize the BP neural network: GA-BP 
network and PSO-BP network. Temperature condition, the retention rate of the rubber compression set, the number of iterations of the neural network and run times were the same as mentioned above. The variance and mean value of MSE of the three models, which characterize the stability and accuracy of the neural network prediction, are shown in Table 3 and Figure 3,

Table 3 Comparison of stability and prediction accuracy of the three neural network models.

\begin{tabular}{|c|c|c|c|}
\hline Network & BP & PSO-BP & GA-BP \\
\hline $\begin{array}{c}\text { Minimum } \\
\text { value }\end{array}$ & 259 & 856 & 2400 \\
\hline $\begin{array}{c}\text { Maximum } \\
\text { value }\end{array}$ & $\begin{array}{c}2.443 \times 10 \\
12\end{array}$ & 2453 & 3375 \\
\hline Average & $8.09 \times 10^{10}$ & 2103 & 2454 \\
\hline Variance & $1.97 \times 10^{23}$ & $3.99 \times 10^{5}$ & $3.03 \times 10^{4}$ \\
\hline $\begin{array}{c}\text { The mean } \\
\text { value of MSE }\end{array}$ & 1.45 & 0.37 & 0.36 \\
\hline
\end{tabular}

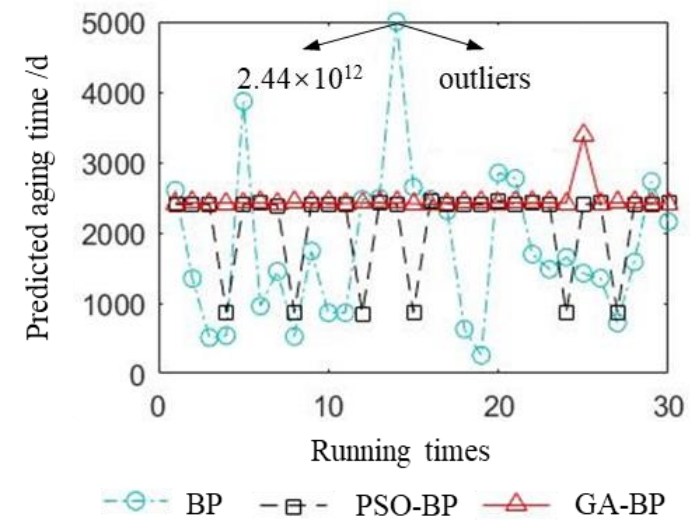

Figure 3 The distribution of predicted aging time with running times for three neural network models

As shown in Table 3 and Figure 3, the prediction results of BP neural network are widely distributed, and its variance is $1.97 \times 10^{23}$, which means that the prediction results are very unstable. The MSE of this network is 1.45 , which indicates that the accuracy is general.

The distribution of predicted aging time for PSO-BP network is relatively centrally. The variance is $3.99 \times 10^{5}$, which means that its stability is much better than BP network. The MSE of it is 0.37 , which indicates that the prediction accuracy is better than BP network.

The predicted aging time of GA-BP network are basically around 2400 , and only 1 point deviates to 3375 and the variance is $3.03 \times 10^{4}$. Its prediction results are very concentrated and stable. The MSE of the network is 0.36 , which indicates that the prediction accuracy is very good.

\subsection{The optimization process and convergence speed}

During above optimization process, the relationship between fitness value of the optimal individual and evolutional generation is shown in Figure 4. The MSE of the first 50 iterations of 100 iterative training process is shown in Figure 5. After 50 iterations, the MSE of three networks has been greatly reduced and their values were very close.

As shown in Figure 4, during the optimization process, PSO algorithm found the best indivisual faster than GA and the convergence speed of PSO-BP was faster than GA-BP and BP during the neural network training process, shown in Figure 5.

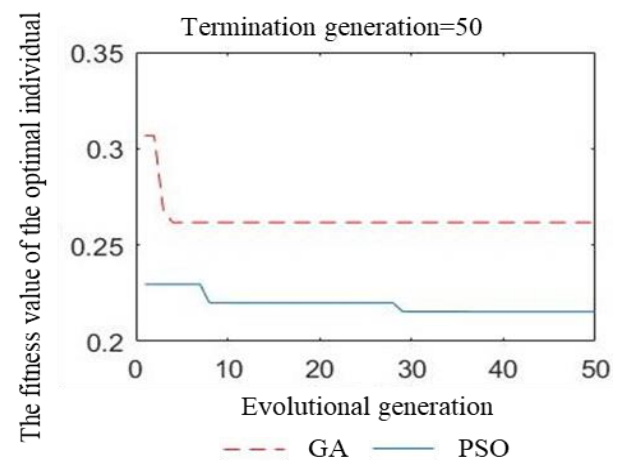

Figure $4 \mathrm{GA}$ and PSO optimization process

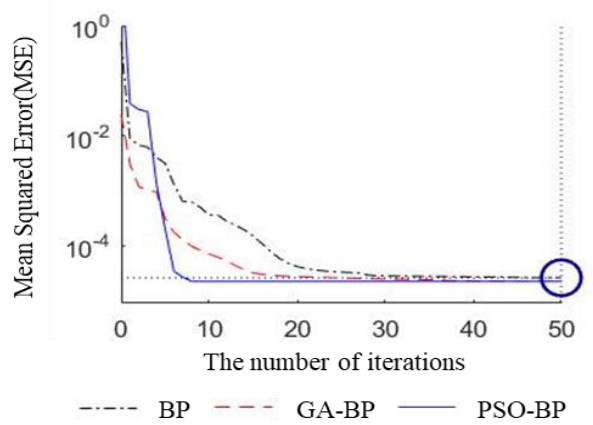

Figure 5 The training process of three networks

Based on above results, the comparison of the stability, prediction accuracy and convergence speed of three models, BP,GA-BP and PSO-BP is shown in Table 4 .

Table 4 The comparison of the stability, prediction accuracy and convergence speed for three models: BP, GA-BP and PSO-BP.

\begin{tabular}{|c|c|c|c|}
\hline Network & Stability & $\begin{array}{c}\text { Prediction } \\
\text { accuracy }\end{array}$ & $\begin{array}{c}\text { Convergence } \\
\text { speed }\end{array}$ \\
\hline BP & Poor & General & Poor \\
\hline GA-BP & Good & Good & General \\
\hline PSO-BP & General & General & Good \\
\hline
\end{tabular}

The GA's crossing and mutation operation is random and has no clear guidance, so the convergence speed is slow. The advantage of GA-BP is that its global search ability is strong. Above reasons make GA-BP more stable. On the contrary, that the optimization process of PSO has clear guidance and its convergence speed was fast made is less stable[10].

In a word, The GA-BP network has better stability and prediction accuracy, although the convergence speed of the PSO-BP is better. The precdiction acuracy is more important for model to be used. Therefore, the GA-BP network was employed to predict the aging life of NBR26 gasket. 


\section{The comparison of prediction result from GA-BP network model, traditional dynamic model and experimental data}

For the traditional dynamic model, the aging time $t$ and the rubber mechanical properties $P$ conform to the following relationship:

$$
P=B \exp \left(-k t^{\alpha}\right)
$$

Where $k$ is the constant of aging rate, and $B$ and $\alpha$ are constants. The relationship between aging rate constant $k$ and temperature $T$ can be expressed by Arrhenius formula:

$$
k=A \exp (-E / R T)
$$

Where $E$ is the activation energy, $R$ is the gas constant, $T$ is the temperature, and $A$ is the constant. The parameters in the formula were determined by linear regression and successive attempt.

The predicted results from traditional dynamic model and GA-BP network model and the experimental data are shown in Figure 6. The relative errors of the prediction results are shown in Figure 7.

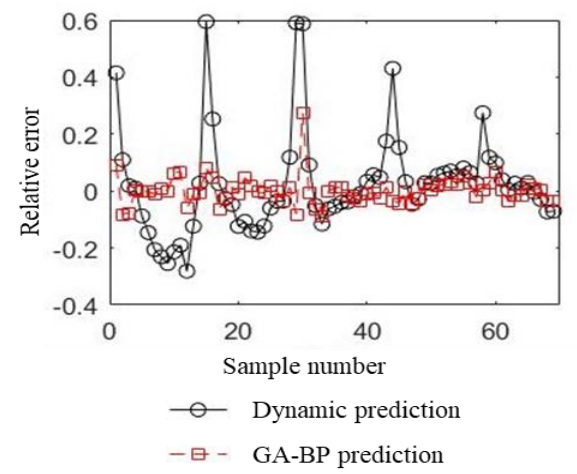

Figure 6 Comparison of prediction results from GA-BP model, traditional dynamic model and experimental data

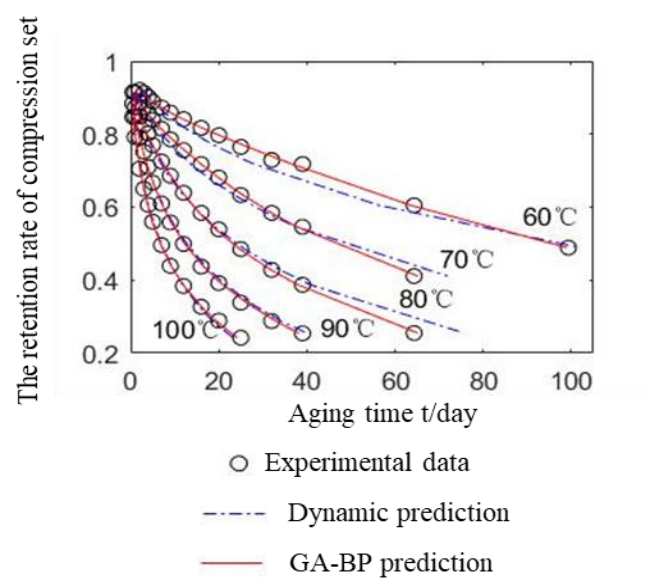

Figure 7 Comparison of relative errors of prediction aging time between traditional dynamic model and GA-BP model.

From Figure 6 and Figure 7, the prediction result of GA-BP model is more consistent with the experimental data, and the relative error of GA-BP model is basically within $10 \%$. However, the prediction error of traditional dynamic method is relatively large. Therefore, the prediction accuracy of GA-BP network model is higher than traditional dynamic model. The results above indicate that the GA-BP model is an more effective and better model for aging life time prediction.

\section{Conclusion}

In this paper, Three neural network methods were studied for predicting the aging life of NBR-26 gasket. In order to obtain stable output results, genetic algorithm and particle swarm optimization were used to optimize BP neural network model established. Stability, accuracy and convergence rate of the three network models were used as evaluation indicators. Finally, the GA-BP network model selectedd was compared with the traditional dynamic model and the experimental data. The conclusions were as follows:

(1) The advantages of artificial neural network prediction model established include multiple input factor parameter effecting rubber aging and no need to consider the complex nonlinear mathematical relationship between input and output, so it can be used to predict the aging life of rubber.

(2) Stability and precision of three neural network algorithms were compared that GA-BP network model was better than PSO-BP and BP network model. As for the convergence speed, the PSO-BP network model was the fastest. Because the accuracy and stability are more important for predicting rubber aging life time, the GABP network model was more suitable and as the selected model.

(3) Comparing with the classical dynamic prediction model, the GA-BP network model has higher prediction accuracy, and is more consistent with the experimental results.

\section{References}

1. X. Xiao, Y.F. Zhao, W. Xu, M.S. Zhan. Aerospace Materials \& Technology,37(1):6-10(2007)

2. Y.J. Li. Rubber Industry, (5):289-296(1994)

3. Pellinen $\mathrm{T} \mathrm{K}$, Witczak $\mathrm{M} \mathrm{W}$, Bonaquist $\mathrm{R} \mathrm{F}$. Engineering Mechanics Division Conference, 83101(2003)

4. A.U. Paeglis. Rubber Chemistry \& Technology, 77(2):242-256(2004)

5. K.T. Gillen, M. Celina. Polymer Degradation \& Stability, 71(1):15-30(2000)

6. Z.F. He. MATLAB R2015b Neural Network Technology, 159-169(2016)

7. J.C. Li, G.J. Qin, X.S. Wen. Journal of Vibration Measurement \& Diagnosis, 22(4):260-264(2002)

8. A Li. Rubber Reference Materials, (4):44-48(2009)

9. X.C. Wang, F Shi, L Yu. Analysis of 43 Cases of MATLAB Neural Network, 20-32:2013.

10. X.Y. Zhang, X.M. Hu, Y Lin. Journal of Frontiers of Computer Science \& Technology, 8(1):90-102(2014) 